\title{
Research on Advertising Marketing in E-sports Competition in China
}

\author{
Xi GUO ${ }^{1}$ \\ ${ }^{1}$ College of Tourism and Aviation Services, Guizhou Minzu University, Guiyang, China
}

\begin{abstract}
With the rapid development of the exhibition industry and the economy, e-sports is undoubtedly one of the most popular exhibition projects nowadays. The rise of E-sports has also led to the development of E-sports advertising marketing. This article is to discuss the advertising marketing in e-sports competition in China and the prospect of the market, especially the advertising strategies used by major brands and the example of League of Legends. It is claimed that it is the era of digitization and precision now. Only by sufficient researching, reasonable technology and conducting social marketing, precision marketing and personalized marketing, can advertising marketing be fully developed. Several suggestions have been put forward in this article.
\end{abstract}

\section{Introduction}

E-sports refers to the sports in which the game of electronic games has reached the level of "competition". The narrow definition of e-sports only refers to the game of e-sports, that is, the online game that can carry out fair confrontation. The broad definition of e-sports mainly includes e-sports competition, e-sports games, e-sports media and so on. E-sports discussed in this paper refers to E-sports in a broad sense. It has two basic characteristics: electronic (game / entertainment) and sports (Sports), which not only meets the needs of fun of the consumers, but also reflects the instinct of human competition. Esports can also be understood as a virtual environment competition, electronic equipment as the device and information technology as the core. Through information technology, a unified competition rule is established, and fairness and antagonism are created in the game. E-sports has become a new sports project. It is a high-tech and digital cultural industry[1]. Because it is in a certain geographical scope, many competitors and spectators compete. It is a group social activity in which relevant personnel gather together, so E-sports competition is also It is a part of the exhibition industry. Convention and exhibition economy is generally regarded as a highincome and high-profit industry. The basic characteristics of e-sports include large user base, rapid growth of market scale and good development prospect. At present, the popularity of e-sports has reached a level that can not be ignored. Therefore, the rise of e-sports has also led to the development of e-sports in advertising marketing. Through data analysis, it could be found that the characteristics of young users in e-sports, as well as the large number of other users, the active consumer market, the consumption ability and high willingness of e-sports users make this industry gradually become an attractive new field exhibition industry. How to choose a good platform and advertising marketing methods to realize the potential and to satisfy the hundreds of millions of users behind the e-sports industry is the top priority of the major brands in the future.

People's consumption level and pursuit of entertainment life created the rapid development of esports industry in the world. Among the top ten products in the PC game market, six are games for e-sports, accounting for nearly $80 \%$ of the game market revenue. China's e-sports industry develops significantly in recent years. However, in China, the proportion of PC's e-sports games is still relatively low, and there is room for the market growth. The number of global e-sports fans is growing rapidly. In 2019, the number of global e-sports spectators has reached 443 million, including 198 million e-sports fans and 245 million temporary spectators.

\section{Analysis of E-sports advertising marketing strategy}

\subsection{Audience analysis}

The audience of e-sports may include the most difficult to reach, but also the most accurate part of the advertising. Therefore, it is very important to understand and grasp the target users first, and try to avoid those advertisements with high frequency. To understand this group, only by truly experiencing as a player, can the advertisers keep up with the changing trend of this group, find out the resonance point, and then design the advertisement that can attract the most players according to their likes and dislikes.

E-sports advertising marketing is different from 
ordinary marketing. The best way to make the advertisement successful is to join the key opinion leader. Learning about games terms as well as the latest information about the game and players and getting a deep understanding of the rules will bring great advantages for the advertising. The following figures can help to analyze the user groups of e-sports.

As can be seen from the figures, the number of e-sports users is increasing year by year, and reached the highest growth rate in 2017 . In addition, among the consumers of e-sports, middle and high consumption groups account for the largest proportion, which indicates that the consumption ability of e-sports consumers is strong. The statistical data confirm the characteristics of e-sports consumption structure and the number of demand side, as well as the huge consumption potential of e-sports market. These changes in consumption level provide a solid foundation for the prosperity of e-sports market.

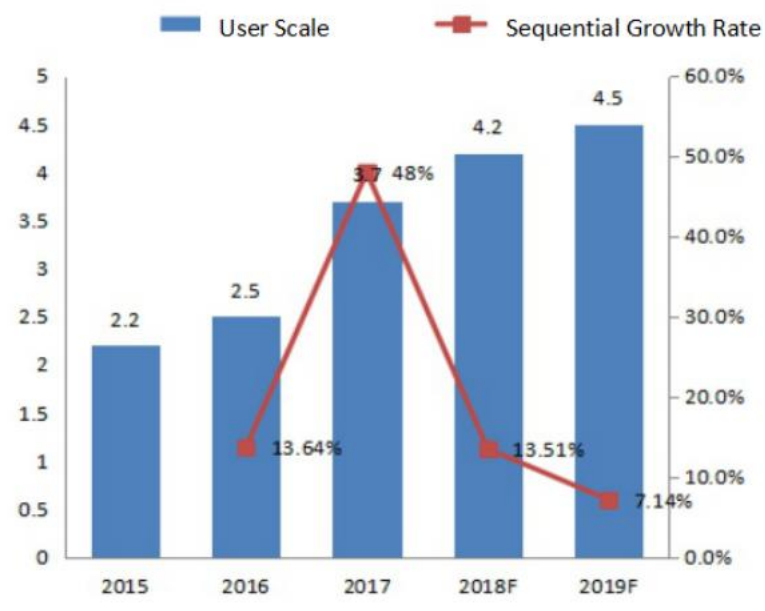

Fig1. the scale of e-sports users

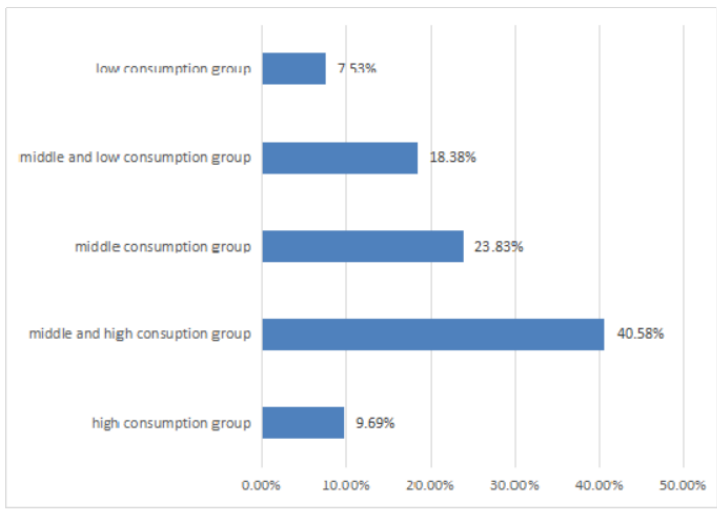

Fig2. The pattern of consumption of the e-sports users

The users of e-sports have obvious characteristics of penetration of Internet aborigines and pan-entertainment. E-sports audiences also prefer entertainment applications. In addition to mainstream entertainment applications, such as live and short video, there is a high level of penetration in animation and vertical video. It is laterally shown that the mutual penetration between e-sports and secondary elements is relatively deep. The social aspect of online gaming and the following discussion of may reinforce the self-esteem of the players as well as the fans, which means a sense of accomplishment to an adolescent's life [2].

E-sports has the characteristics of sports competition and digital games, and is affected by the market capacity. The economic environment of e-sports consumption has been a gratifying trend. The market capacity, the strong correlation of digital games and the expansion of e-sports industry make a significant increase in product development, market potential, user groups and even social influence.

\subsection{Moderate marketing}

Frequent advertising information will cause the disgust of the audience, especially game players, and most of them are young men who are usually hard to reach by brand marketing. To attract the attention of these players, the most important thing is to show what they can see directly without disturbing them in the game or in the game. Therefore, it is necessary to combine advertising with things that can attract their attention in the beginning, such as the advertising banner at the beginning of an article, and the buffer advertisement at the end of the video, which will not disturb their attention during the game. In addition, the video can also be named, and the original advertisement can often get better results by displaying it to game players in a subtle way.

\subsection{Platform selection}

Understanding the audience's likes and dislikes is only half done, but also needs to know where these users gather. Overseas, the live game platform twitch has attracted a large number of game players, becoming a huge and professional e-sports community, where players play games and watch live games. In 2017, consumers spent the longest time watching "League of Legends" on the twitch platform, with a cumulative duration of more than 1 billion hours. In China, Betta TV is a live broadcast sharing website with bullet screen style, providing users with video and event live services. Betta TV mainly focuses on game live broadcast, covering entertainment, variety show, sports, outdoor and other live content. Panda live broadcast is a bullet screen video live broadcast website founded by Shanghai Panda mutual Entertainment Culture Co., Ltd. The content of panda TV will be more pan-entertainment in addition to game live broadcast. It will provide users with live broadcast of concerts, sports events and other forms. As of 2016, Huya live broadcast has 210 million registered users, including 96 million monthly active users and more than 150 million monthly revenue. User stickiness and platform interaction are maintained at a very high level, with daily average viewing time of 135 minutes. As for another platform, Penguin e-sports is Tencent's largest mobile e-sports platform. Its positioning is the "connector" of e-sports ecology, integrating the resources of Tencent, QQ mobile game and Tencent mutual entertainment team. They cooperate with professional e-sports events such as QGC and TGA deeply. At the same time, it gathers a large number of big brand game anchors, which integrates professional competition of mobile game, interactive video live broadcast and game gift bag sharing, so as to provide one-stop mobile e-sports experience for player 
users. Therefore, many advertisers are turning to key opinion leader and players to promote marketing.

As long as the right communication platform is chosen and the right voice is spread, fans will be more likely to pay attention to the e-sports competition and the effect of the advertisement would be better. The survey found that more than half of the video content watched by these esports fans came from the mobile platform, so the media strategy of brand companies must match the consumption habits of the audience. Suitable game media platforms include vertical media such as Penguin video games, YouTube gaming and twitch, while platforms such as panda TV, Betta TV, snapchat and instagram can be used as media channels for the general public. At the same time, it is different from SNS, youtube, INS, microblog and other websites. There is no place in the live platform where the audience can express their emotions. The transmission of live broadcast information is one-way one-to-many. Mobile live broadcast has more real-time interaction than traditional TV broadcast media, but it is limited to the broadcast process. After the live broadcast, there was no emotional expression or communication. Therefore, the effect of mobile live broadcasting on the retention rate is poor. The advertising effect of Wechat has always been good, mainly because of the close network based on friends and acquaintances, large number of users, high retention rate, more clicks, and increased number of ads, so the marketing effect is very good. Live apps can only gather popularity at the start of the live broadcast. After the live broadcast, the audience will separate, which makes the afterheat effect of the live broadcast dissipate quickly and can not arouse the audience's emotional resonance. The real-time live broadcast platform itself is difficult to be compatible with user fragmentation habits, and can not be completely upgraded to social scenarios. The unfamiliar relationship between the audience is not conducive to emotional communication.

\subsection{Comprehend the symbiosis between brand and e-sports}

Under the circumstance of new pan-entertainment marketing, the maximization of brand communication volume and the improvement of conversion rate reflect the characteristics of win-win, which is called "symbiosis" in biology. However, symbiosis is not limited to the biological field. Complex economic entities can not completely distinguish their respective boundaries, and interact with each other through some material media, forming a symbiotic relationship of interdependence and cooperation.

In the field of pan entertainment marketing such as esports, symbiosis has become more and more marketing mode and exploration trend. For example, the advertising and marketing activities of Meituan which is a take-out platform and the game Player Unknown's Battle Grounds in 2019 are not only the spread of Meituan's food, but also the spread of the game since the Chinese players all call the game "Chiji" meaning eating chicken in the Chinese language and then the there were sound effects of the advertising in Meituan. Both of them have become the beneficiaries of the activities. In this pan-entertainment marketing, symbiosis and win-win, fans exchange, passion, together contribute to the conversion rate.

For the brand owners who decide to carry out e-sports marketing, it is necessary to think about how to deeply cultivate their own e-sports ecology, and combine their own brand connotation, brand tonality, consumer groups with e-sports to produce better scenes and products. Meanwhile, managing the shortages of products exposed and conversion is needed as well.

According to the symbiosis theory, the increase of brand marketing will certainly provide a stronger and more comprehensive industrial chain for the sunrise industry of e-sports, and become a greater industry potential energy. At the same time, in the cognition of esports and the spread of e-sports culture, advertising will permeate into the corner of the society to enhance the recognition and recognition of this emerging project. How to select the right brand partner and how to explore new ways of cooperation are also the focus of e-sports industry.

\section{Analysis on the development trend of advertising marketing in e-sports Taking the League of Legends as an example}

It is widely acknowledged that the revenue of e-sports mainly comes from the sponsorship, the sale of broadcasting rights and users' payment to watch the competition lively on the platforms. As a result, they account for 77.1 percent of the total revenue, while ticket and derivative revenues constitute 15.3 percent. Thus, the upgrading of the e-sports business model is urgently needed to meet the potential of the e-sports further value [3]. Among the strategy of this upgrading, the advertising marketing plays a significant role.

In all the "League of Legends" competition, the "League of Legends" global finals is the most authoritative competition and has been held for 9 times. This paper will take it as a case study to analyse the development of esports advertising marketing.

\subsection{Thinking of new ideas of derivative IP + e- sports marketing}

IP means "intellectual property", and brand IP refers to "brand intellectual property" derived from the concept of intellectual property. Shaping brand IP is to excavate brand self. The value of body and the pursuit of cultural identity are also the process of emotional brand. In terms of IP and e-sports, the content of the entire ecological level is still built around the users. In 2017 years, the League of Legends launched the hero alliance universe, and the updated contents include Demacia, Zuan city-state information, etc. In the past, there was no world view. Now, after the establishment, more specific and rich content can be seen and the hero's story and their changes can be better understood. In the future, there would be more stories or animation about heroes based on the world outlook.

Interactive marketing needs deep insight into 
consumers' interests and concerns in e-sports competition, and then attract consumers' attention, so as to encourage consumers to actively participate in the interaction and form an invisible secondary communication, so that consumers can participate in brand hot spots together. New brand culture elements constitute a new brand IP, thus enriching the connotation of brand IP, enhancing brand culture, and realizing product upgrading. Therefore, it can be seen that a key to the success of e-sports interactive marketing in advertising marketing is that the brand side has been creating its brand IP together with consumers. The brand IP marketing strategy of e-sports is mainly interactive marketing and cross-border marketing, The so-called interactive marketing is to create brand IP together with consumers and gain insight into consumers Demand, attract consumers to have in-depth interaction with them, so as to aggregate fans. And the cross-border camp marketing can build its brand into an emotional brand IP.

Through cross-border and cross variety cooperation, the brand side creates a unique cultural symbol of the esports competition, establishes a brand IP with emotion, and integrates the brand IP with this emotional attribute into the brand culture and transmits it among the audience of the e-sports competition, so as to make the brand IP constantly integrate into the characteristics of electronics and competition. Through the establishment of brand IP, the brand side breaks away from the original unique label and extends to more category fields. Constantly expand their own brand value, maintain the brand's continuous vitality, and realize the boundless implantation of brand IP.

\subsection{Industrial linkage integrated marketing}

The official partners of the League of Legends in the S7 season of the global finals are five international brands in different fields: Mercedes Benz, Yili Guliduo, L'Oreal men, Logitech $\mathrm{G}$ and Intel. In addition to the joint creative posters released by the partners on the same day, the five partners not only carried out in-depth brand placement exposure during the live broadcast of the competition, but also carried out in-depth brand implantation exposure from the aspects of brand voice, regional marketing, exhibition area construction, creative investment and node linkage. In every key marketing node, it could be seen that the simultaneous linkage of the five brands had become a unique ceremony of S7. From the perspective of marketing effect, the vision of brand linkage has full impact. In addition to the linkage in marketing, the five major brands have jointly opened a large-scale e-sports ecommerce festival on the e-commerce platform Jingdong, with the S7 schedule as the node.

Brand linkage has full visual impact. This competition is the first cooperation of five international brands in the field of marketing, and also the first International competitions, e-sports products, multiple international brands and e-commerce have been realized at one time. From the marketing strategy of S7 games, we can use the way of industrial integration in the brand in terms of production voice, regional marketing, exhibition area construction, creative investment and node linkage.

\subsection{Pan-entertainment based on fan economy}

"League of Legends" global finals S7 games, according to statistics, the total watching time has exceeded 1.2 billion hours. According to official statistics, each game in the S8 season attracted an average of 47 million spectators worldwide, of which $98 \%$ were Chinese.

The development of e-sports depends on the game industry and integrates the industry attributes of "big sports" and "pan- entertainment". Its core is to attract fans' attention through e-sports competition, so as to develop and cultivate business value. In the e-sports industry, the development space is determined by the quality of e-sports events and the scale of fans' interaction. The holding of the e-sports competition realizes the centralized exposure of the brands and expands the influence of the brands. What is more, compared with mass marketing, there are obvious differences in the characteristics of the audience, the mood of the audience and the form of marketing activities. Esports enthusiasts are often people who actively spend money to "buy advertising" in the process of purchasing their favorite e-sports products. Since the sponsor logo of e-sports team and the surrounding products of sponsors have been exposed to the consumption of the fans for many times, the fans would not dislike them.

\section{Conclusion}

Through the detailed analysis of the domestic E-sports advertising marketing strategy in China, there are still some deficiencies and need to be improved. Based on the analysis of the development trend of domestic e-sports advertising marketing, this paper analyzes the existing advertising marketing strategies of domestic e-sports. It has been shown that brand owners have understood the characteristics of the target users, as well as the huge potential of them to some extent. However, it is still need to be researched where these users are gathered. Then it is supposed to be thought about how to deeply cultivate the e-sports ecology in this country, and combine the brand connotation, brand tonality, consumer groups and e-sports to better produce the scenes and products. From the development trend of e-sports in recent years, this paper finds out the existing trends of e-sports advertising marketing, including four major trends: opening up new ideas of derivative IP + e-sports marketing, industrial linkage integrated marketing, pan entertainment based on fan economy and e-sports AI, hoping to bring certain reference value for improving the advertising marketing of domestic e-sports technology in China.

\section{References}

1. Liu Xinwei, “China's e-sports industry displays its strength", China's Foreign Trade, vol.5, p70-71, 2019.

2. Harri Jalonen, "The Value of E-Sports Is in the Eye of the Beholder, But Can E-Sports Operators Influence What the Spectators See?" , Advances in Applied Sociology, vol.7, 2019.

3. Zhang Shasha, "Sports on screen", Beijing Review, vol.28, p.38-39, 2019. 
4. Liang Yanjie, "Scene advertising marketing of network live broadcast", New Media Research, vol.15, p.43-44, 2018.

5. Pan Xiaoyu, "The application strategy of big data in advertising marketing", Northern Economy, vol.10, p55-56, 2018.

6. Chu Meng, "From the Gilded Age to the Golden Age", China Advertising, vol.10, p76-79, 2019.

7. Wang Lin, "New way of E-Game Marketing”, China Advertising, vol.10, p83-88, 2019. 